\title{
The effect of sitagliptin on cardiovascular risk profile in Korean patients with type 2 diabetes mellitus: a retrospective cohort study
}

This article was published in the following Dove Press journal:

Therapeutics and Clinical Risk Management

15 March 2016

Number of times this article has been viewed

\section{Sooyoung Shin' \\ Hyunah Kim²}

'College of Pharmacy, Ajou University, Suwon, ${ }^{2}$ College of Pharmacy, Sookmyung Women's University, Seoul, Republic of Korea
Correspondence: Sooyoung Shin

College of Pharmacy, Ajou University, 206, World cup-ro, Yeongtong-gu, Suwon 16499, Gyeonggi-do, Republic of Korea $\mathrm{Tel}+823$ I 2193456

Fax +82 31 2193435

Email syshin@ajou.ac.kr
Background: A 2013 postmarketing study suggested a possible link between saxagliptin use and hospital admission for heart failure. Cardiovascular (CV) effects of sitagliptin, the most commonly prescribed antidiabetic in the same class as saxagliptin, have not been evaluated much in Asian patients with type 2 diabetes. This study sought to ascertain the CV safety of sitagliptin in Korean patients.

Methods: A retrospective cohort study of 4,860 patients who were classified into the sitagliptin and metformin groups was conducted using electronic patient data retrieved from a major tertiary care medical center in Korea. Primary composite end points included CV death, myocardial infarction, and ischemic stroke. Secondary composite end points included the aforementioned individual primary outcomes plus hospitalization due to unstable angina, heart failure, or coronary revascularization. A Cox proportional-hazards model was used to compare CV risk associated with drug exposure. Results: Following propensity score (PS) matching in a 1:2 ratio, 1,620 patients in the sitagliptin group and 3,240 patients in the metformin group were identified for cohort entry. The PS-matched hazard ratio (HR) and 95\% confidence interval (CI) for sitagliptin relative to metformin were, respectively, 0.831 and $0.536-1.289(P=0.408)$ for primary end point and 1.140 and $0.958-1.356(P=0.139)$ for secondary end point. Heart failure hospitalization rates did not differ significantly between the two groups, with the PS-matched HR of 0.762 and $95 \%$ CI of 0.389-1.495 $(P=0.430)$. When only those patients at high risk of ischemic heart disease were included for analysis, no excess $\mathrm{CV}$ risk was observed with sitagliptin compared with metformin. Overall, there were no substantial between-group differences in rates of adverse events, such as hypoglycemia and incident pancreatic disease.

Conclusion: Sitagliptin was not associated with elevated risk of CV complications including myocardial infarction, ischemic stroke, heart failure, and coronary revascularization, compared to metformin therapy among Korean patients with type 2 diabetes.

Keywords: sitagliptin, dipeptidyl peptidase 4 inhibitors, cardiovascular outcomes, type 2 diabetes

\section{Introduction}

Patients with type 2 diabetes mellitus (T2DM), especially those who have elevated levels of fasting plasma glucose $(\mathrm{FPG})$ or hemoglobin $\mathrm{A}_{1 \mathrm{c}}\left(\mathrm{HbA}_{1 \mathrm{c}}\right)$, are at increased risk of cardiovascular $(\mathrm{CV})$ morbidity and mortality. ${ }^{1-3}$ Therefore, the treatment of T2DM has been centered on the goal of achieving and maintaining glycemic control without sacrificing patient safety and tolerability.

In the late 2000 s, concerns have been raised with respect to risk of cardiac adverse events associated with the use of oral antidiabetics (OAs), ${ }^{4-6}$ most notably rosiglitazone 
which is a thiazolidinedione originally approved by the US Food and Drug Administration (FDA) in 1999. ${ }^{7-9}$ It is well established that improved regulation of glycemic levels contributes to reducing the risk of diabetes-induced microvascular complications commonly experienced by patients with type 2 diabetes. ${ }^{10,11}$ However, conclusive evidence on the risk of macrovascular complications, most notably $\mathrm{CV}$ events which are the leading cause of mortality in patients with diabetes, remains elusive. In 2008, the controversy over the CV safety profile of diabetes therapy ultimately prompted the FDA to require all novel therapeutic options for diabetes to establish long-term $\mathrm{CV}$ effects as part of pre- and post-approval commitments. ${ }^{12}$

In accordance with the FDA guidance, three postmarketing clinical trials of dipeptidyl peptidase 4 (DPP-4) inhibitors have been conducted to investigate $\mathrm{CV}$ risks of those newly available class of OAs. ${ }^{13-15}$ The randomized controlled trials (RCTs) showed that the individual study agent provided statistically neutral effects on the rates of $\mathrm{CV}$ events relative to placebo. The SAVOR-TIMI 53 trial and a subsequent 2015 meta-analysis, however, indicated a possible association between the DPP-4 inhibitor and a higher incidence of hospital admission due to heart failure..$^{14,16}$

On the contrary, previous meta-analyses showed that DPP-4 inhibitors might lower the incidence of major adverse $\mathrm{CV}$ events compared with a placebo or other hypoglycemic agents. ${ }^{17-19}$ A more recent population-based cohort study using the US administrative health insurance claims database, however, failed to detect any evidence that DPP-4 inhibitors are associated with either an increased or a decreased risk of CV diseases (CVDs) in patients with type 2 diabetes. ${ }^{20}$ Taken together, study results to date suggest that the potential risk of adverse cardiac outcomes indicated in the SAVOR-TIMI 53 study is presumably not a class effect of DPP-4 inhibition. Nonetheless, uncertainty remains as to whether individual DPP-4 inhibitors are safe from the CV standpoint. Thus far, without definitive proof of how DPP-4 inhibition affects patient CVDs, the glucose-lowering therapy has been commonly prescribed as monotherapy or as combination therapy with other hypoglycemic agents to combat T2DM.

In light of conflicting evidence, further investigation is warranted to evaluate whether differential risk of CVDs exists among DPP-4 inhibitors. Of particular importance is the CV safety profile of sitagliptin, the first approved and most commonly prescribed agent in clinical practice settings within the category of medications. ${ }^{21}$ The only RCT conducted to date to investigate $\mathrm{CV}$ outcomes of sitagliptin found that adding sitagliptin to standard care was not associated with excess risks of $\mathrm{CV}$ events during a median follow-up of
3 years. ${ }^{15}$ However, the antidiabetic in question was not compared head to head against metformin therapy which is the mainstay first-line antidiabetic in real-world clinical settings; hence, external validity of the study may have to be interpreted within the context of RCT. Additionally, the dominant ethnic group enrolled in the RCT was White (with only $22 \%$ Asians); therefore, patient characteristics may not be representative of Asian patient populations. Growing evidence suggests that the pathophysiology of T2DM and the efficacy of DPP-4 inhibitors in diabetes management may differ between Asians and other ethnic groups. ${ }^{22}$ Thus, there remains a clinical need to verify the safety of the most commonly prescribed DPP-4 inhibitor in diabetic patients with Asian ethnic background. In this study, we performed a retrospective cohort study using patient data retrieved from institutional medical records to evaluate the $\mathrm{CV}$ effects of sitagliptin compared against those of metformin therapy among Korean patients with T2DM.

\section{Methods}

\section{Study design and cohort}

The protocol of this retrospective observational cohort study was approved by the Institutional Review Board of Ajou University Hospital (AJIRB-MED-EXP-14-221). Patient data were collected from electronic medical records of the study institution of a 1,108-bed tertiary care, university-affiliated, teaching hospital in Korea. Informed consent from study patients was not required as patient records were de-identified and anonymized prior to data analysis. Adult patients, aged 18 years and older, with a history of T2DM (identified by the International Classification of Disease, Tenth Revision [ICD-10] codes E11-14) were initially selected for the study cohort if they had received the study medications of sitagliptin or metformin between January 1, 2010 and December 31, 2014 , with a therapy duration of at least 6 consecutive months. The consecutive therapy was defined as when a study medication order occurred within 1.5 times the days supply per prior prescription following its end date.

Two treatment groups were defined as follows: 1) patients treated with sitagliptin combined with other OAs (sitagliptin group) and 2) patients treated with metformin plus non-DPP-4 inhibitors (metformin group). The mutually exclusive intervention groups were contrived to account for the reimbursement eligibility criteria of the Korean National Health Insurance plan which recommends and covers metformin as first-line for T2DM, whereas a DPP-4 inhibitor only as add-on to metformin or other OAs. Patients were ineligible for the study cohort if they had a history of any of 
the following: end-stage renal disease, dialysis treatment, estimated glomerular filtration rate $<30 \mathrm{~mL} / \mathrm{min} / 1.73 \mathrm{~m}^{2}$, renal transplantation, or any type of cancer. Patients who received insulin, incretin-based therapy including DPP-4 inhibitors (except sitagliptin), or thiazolidinediones either during the 6-month baseline period prior to cohort entry or at any time during the study period were also excluded from further analysis. All other therapies for the management of diabetes and $\mathrm{CV}$ comorbid conditions were permitted. Of the collected eligible cases, patients were then matched in a 1:2 ratio to the sitagliptin or metformin groups using propensity score (PS) matching per age, sex, weight, body mass index (BMI), duration of diabetes, atherosclerotic disease, hypertension, dyslipidemia, prior ischemic heart disease, prior heart failure, and $\mathrm{HbA}_{1 \mathrm{c}}$ level at baseline.

The index date for both groups was determined as the first date of initiating the primary study agent (sitagliptin or metformin) with concurrent use of other glucose-lowering therapy. Patient follow-up began on the index date until the earliest occurrence of any of the following censoring outcomes: a follow-up discontinuation, any of the end-point CV events, the end of the study period (December 31, 2014), or death.

Additionally, those patients who met the following high-risk criteria were identified for a subgroup analysis: 1) patients with a history of an ischemic CV event induced by atherosclerosis in the coronary or cerebrovascular system prior to cohort entry and 2) male patients of at least 55 years old or female patients aged 60 years or older, with extra risk factors including dyslipidemia, atherosclerotic disease, hypertension, and active smoking status.

\section{Study outcomes}

The primary $\mathrm{CV}$ outcome was a composite of $\mathrm{CV}$ death, myocardial infarction (MI), or ischemic stroke. The end-point events were captured via hospital admission or an emergency department visit with a CVD diagnosis identified with ICD-10 codes (CV death: I20-70; MI: I21-23; or ischemic stroke: I63, I65, and I66). The secondary end points included individual outcomes evaluated as the composite primary outcome along with other end points including hospitalization due to unstable angina (I20), heart failure (I50), or coronary revascularization (identified with procedure codes for percutaneous coronary intervention or coronary artery bypass grafts). The outcome date was determined as the earliest date a patient experienced a given outcome event. Admission to hospital with a primary diagnosis of hypoglycemia along with any episodes of blood glucose level $<54 \mathrm{mg} / \mathrm{dL}$ during the follow-up period was also identified for safety assessment.
Additionally, any cases with documented serum creatinine level $>6 \mathrm{mg} / \mathrm{dL}$, new-onset renal disease, and incident pancreatitis or pancreatic cancer were also separately captured as an adverse safety event.

\section{Covariates}

The prespecified covariates are as follows: age, weight, and BMI at the index date, sex, duration of diabetes, presence of comorbidities, status of smoking and obesity, levels of $\mathrm{HbA}_{1 \mathrm{c}}, \mathrm{FPG}$, and serum creatinine, and concomitant use of pharmacologic therapies for diabetes and CVD control. The duration of diabetes was defined as the time from the first date with a diagnosis code for diabetes recorded in the institutional electronic medical record until the index date. The following comorbid conditions which existed prior to the index date were identified by ICD-10 codes: atherosclerotic disease, hypertension, dyslipidemia, prior ischemic heart disease, prior heart failure, and renal disease. Concurrent medications used for the management of diabetes and CVDs were also assessed, including metformin, sulfonylureas, DPP-4 inhibitors, glucosidase inhibitors, meglitinides, calcium channel blockers, beta blockers, loop diuretics, thiazides, angiotensinconverting enzyme inhibitors, angiotensin receptor blockers, antiplatelets, statins, and warfarin.

\section{Statistical analysis}

We calculated incidence rates along with $95 \%$ confidence intervals (CIs) of clinical end points for the two exposure groups. The primary and secondary end-point analyses were performed with the use of a Cox proportional-hazards model, and a $P$-value of $<0.05$ was considered to be statistically significant. To balance potential between-group confounders, PS matching was applied with the nearest neighbor matching method at a fixed ratio of 1:2 (two controls per one study patient). A multivariable logistic regression model with the baseline variables as covariates was used to estimate the PS for each patient, which predicts the probability of patient exposure to sitagliptin versus metformin therapy given prespecified baseline variables. Kaplan-Meier curves were plotted for the cumulative hazard of primary and secondary end points in the PS-matched sitagliptin and metformin cohorts. Statistical analyses were performed using SAS 9.4 Software (SAS Institute Inc., Cary, NC, USA).

\section{Results \\ Study patients}

During the 5-year study period, there were 5,141 patients with diabetes who satisfied the eligibility criteria of the study. 
Following PS matching with a 1:2 ratio, a total of 4,860 patients were finally captured for cohort entry, including 1,620 in the sitagliptin group and 3,240 in the metformin group. The baseline characteristics of the PS-matched patients are shown in Table 1. The mean age of the included patients was 56.8 years in the sitagliptin group and 56.5 years in the metformin group, and $42.2 \%$ of patients in each group were female. The mean weight was 67.8 and $67.3 \mathrm{~kg}$ in the sitagliptin and metformin groups, respectively. The median duration of diabetes was 3.6 years (with interquartile range of 2.1-7.0 years) and 3.5 years (with interquartile range of 1.7-7.2 years) in the sitagliptin and metformin groups, respectively. Comorbid conditions, such as atherosclerotic disease, hypertension, dyslipidemia, prior ischemic heart disease, prior heart failure, and renal disease, were similarly distributed between the groups. Overall, baseline characteristics were well balanced in the PS-matched groups. The median follow-up duration was 2.9 years with the interquartile range

Table I Baseline characteristics of the study patients ( I:2 propensity score-matched sitagliptin and metformin cohorts)

\begin{tabular}{|c|c|c|}
\hline Characteristic & Sitagliptin $(N=1,620)$ & Metformin $(\mathrm{N}=3,240)$ \\
\hline \multicolumn{3}{|l|}{ Age } \\
\hline Mean \pm SD (years) & $56.8 \pm 12.3$ & $56.5 \pm 12.2$ \\
\hline$\geq 75$ years, $\mathrm{n}(\%)$ & $124(7.7)$ & $240(7.4)$ \\
\hline \multicolumn{3}{|l|}{ Sex } \\
\hline Female, n (\%) & $683(42.2)$ & $\mathrm{I}, 368(42.2)$ \\
\hline Male, n (\%) & $937(57.8)$ & $\mathrm{I}, 872(57.8)$ \\
\hline \multicolumn{3}{|l|}{ Weight } \\
\hline Mean \pm SD (kg) & $67.8 \pm 6.9$ & $67.3 \pm 7.2$ \\
\hline Body mass index, mean $\pm \mathrm{SD}\left(\mathrm{kg} / \mathrm{m}^{2}\right)$ & $25.0 \pm 1.9$ & $25.0 \pm 2.0$ \\
\hline \multicolumn{3}{|l|}{ Duration of diabetes (years) } \\
\hline Median & 3.6 & 3.5 \\
\hline Interquartile range & $2.1-7.0$ & I.7-7.2 \\
\hline Atherosclerotic disease, $\mathrm{n}(\%)$ & $9(0.6)$ & $19(0.6)$ \\
\hline Hypertension, n (\%) & $562(34.7)$ & $\mathrm{I}, \mathrm{III}(34.3)$ \\
\hline Dyslipidemia, n (\%) & $356(22.0)$ & $734(22.7)$ \\
\hline Prior ischemic heart disease, $\mathrm{n}(\%)$ & $199(12.3)$ & $362(11.2)$ \\
\hline Prior heart failure, n (\%) & $32(2.0)$ & $5 \mathrm{I}(1.6)$ \\
\hline Renal disease, n (\%) & $13(0.8)$ & $21(0.7)$ \\
\hline Smoking, n (\%) & $134(8.3)$ & $306(9.4)$ \\
\hline Obesity, n (\%) & $39(2.4)$ & $81(2.5)$ \\
\hline \multicolumn{3}{|l|}{ Hemoglobin $A_{I c}$} \\
\hline Mean \pm SD (\%) & $8.0 \pm 1.5$ & $7.7 \pm 1.5$ \\
\hline \multicolumn{3}{|l|}{ Distribution, n (\%) } \\
\hline$<6.5 \%$ & $165(10.6)$ & $633(19.9)$ \\
\hline $6.5 \%-<9.0 \%$ & $1,049(67.3)$ & $2,026(63.8)$ \\
\hline$\geq 9.0 \%$ & $344(22.1)$ & $515(16.2)$ \\
\hline Fasting plasma glucose, mean $\pm \mathrm{SD}(\mathrm{mg} / \mathrm{dL})$ & $166.3 \pm 64.2$ & $157.0 \pm 58.8$ \\
\hline Creatinine level, mean $\pm S D(m g / d L)$ & $0.9 \pm 0.5$ & $0.9 \pm 0.4$ \\
\hline \multicolumn{3}{|l|}{ Type of oral hypoglycemic drugs, n (\%) } \\
\hline Metformin & $\mathrm{I}, 067(65.9)$ & $3,240(100)$ \\
\hline Sulfonylureas & $306(18.9)$ & $560(17.3)$ \\
\hline DPP-4 inhibitors & $\mathrm{I}, 620(100.0)$ & $0(0.0)$ \\
\hline Glucosidase inhibitors & $15(0.9)$ & $61(1.9)$ \\
\hline Meglitinides & $5(0.3)$ & $19(0.6)$ \\
\hline \multicolumn{3}{|l|}{ Other medications, $\mathrm{n}(\%)$} \\
\hline Calcium channel blockers & $736(45.4)$ & $1,315(40.6)$ \\
\hline Beta blockers & $391(24.1)$ & $698(21.5)$ \\
\hline Loop diuretics & $119(7.3)$ & $219(6.8)$ \\
\hline Thiazides & $221(13.6)$ & $465(14.4)$ \\
\hline ACEI/ARB & $877(54.1)$ & $1,604(49.5)$ \\
\hline Antiplatelet & $885(54.6)$ & $1,609(49.7)$ \\
\hline Statin & $930(57.4)$ & $\mathrm{I}, 758(54.3)$ \\
\hline Warfarin & $4 \mid(2.5)$ & $60(1.9)$ \\
\hline
\end{tabular}

Abbreviations: SD, standard deviation; DPP-4, dipeptidyl peptidase 4; ACEl, angiotensin-converting enzyme inhibitor; ARB, angiotensin receptor blocker. 
of 1.5-4.6 years and the maximum follow-up period of 5.1 years. The total person-time observed was 5,027 personyears and 9,083 person-years in the sitagliptin and metformin groups, respectively.

\section{CV end points}

During the follow-up period, a primary composite end-point event of CV death, MI, or ischemic stroke was encountered in 29 patients in the sitagliptin group (incidence rate of 5.82 per 1,000 person-years) and in 64 patients in the metformin group (incidence rate of 7.11 per 1,000 person-years). The PS-matched hazard ratio (HR) and 95\% CI were 0.831 and $0.536-1.289$, with a $P$-value of 0.408 . A secondary composite end-point event of CV death, MI, ischemic stroke, plus hospitalization for unstable angina, heart failure, or coronary revascularization was experienced by 206 patients (incidence rate of 44.10 per 1,000 person-years) and 334 patients (incidence rate of 39.08 per 1,000 person-years) in the sitagliptin and metformin groups, respectively. The PS-matched HR was 1.140 , with $95 \% \mathrm{CI}$ of $0.958-1.356(P=0.139)$. The results of incidence rates and PS-matched hazard analysis associated with each component of primary and secondary composite end points are summarized in Table 2. Incidence rates for hospital admission for heart failure did not differ significantly between the two groups ( 2.51 versus 3.33 per 1,000 personyears, respectively), with the PS-matched HR of 0.762 and 95\% CI of 0.389-1.495 ( $P=0.430$ ). Sitagliptin did not appear to increase the risk of any of the individual CV end-point events. Figures 1 and 2 represent the Kaplan-Meier curves comparing the cumulative hazard of primary and secondary end points, respectively. According to log-rank test, no statistically significant difference in the risk of CVDs was noted between the two treatment groups in both analyses.

In the subgroup analysis, high-risk patients were identified based on the existence of CVD or additional CV risk factors prior to cohort entry and evaluated for $\mathrm{CV}$ effects of therapy exposure with analogous methods used in the initial assessment. A total of 517 patients and 1,055 patients were included in the study and control groups, respectively, for the high-risk group analyses. During the follow-up period, 12 composite primary events occurred with 1,563 personyears of follow-up in the sitagliptin group (incidence rate of 7.68 per 1,000 person-years) and 31 composite CVD primary events with 2,832 person-years of follow-up in the metformin group (incidence rate of 10.95 per 1,000 person-years). The PS-matched HR $(95 \% \mathrm{CI})$ was $0.725(0.372-1.413)$ with a $P$-value of 0.346 . A composite secondary end-point event was established in 65 patients in the sitagliptin group (incidence

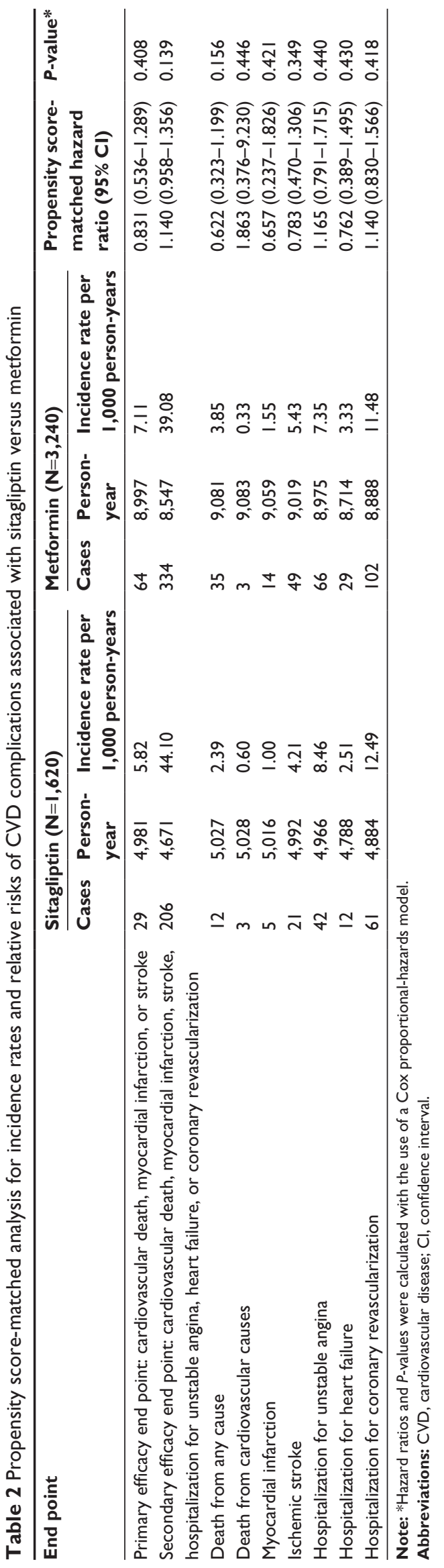




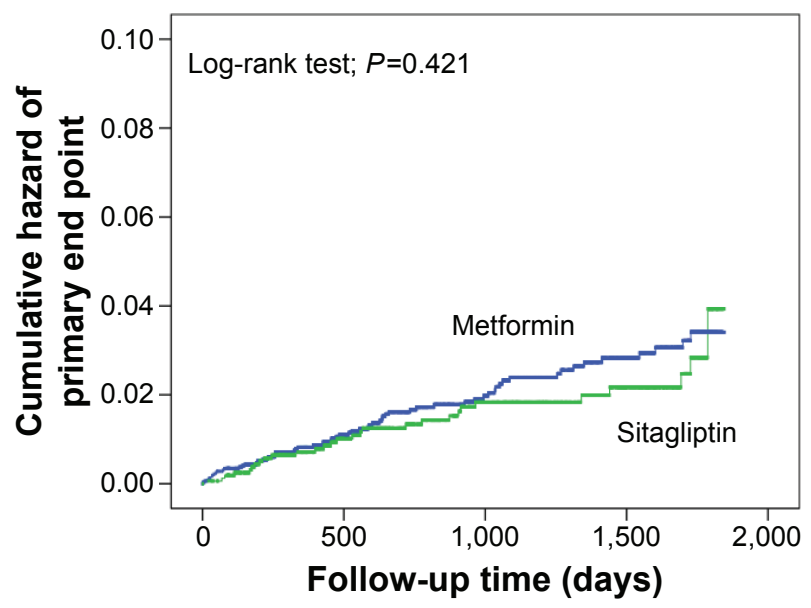

Figure I Kaplan-Meier curves for cumulative hazard of a primary composite end point.

Note: Sitagliptin and metformin cohorts are propensity score-matched.

rate of 45.85 per 1,000 person-years) and in 122 patients in the metformin group (incidence rate of 46.93 per 1,000 person-years). The PS-matched HR (95\% CI) was 1.010 (0.747-1.365), with a $P$-value of 0.949 . Table 3 shows the analysis results of individual components of the composite end points. Incidence rate for hospitalization for heart failure was not significantly different between the two groups, and the PS-matched HR $(95 \% \mathrm{CI})$ was $0.673(0.283-1.602)$, with a $P$-value of 0.370 .

\section{Safety end points}

Individual components of the prespecified safety end points are summarized in Table 4. Hypoglycemic events and pancreatic disease developed sparsely, but the rates appeared numerically more frequent in the sitagliptin group albeit with

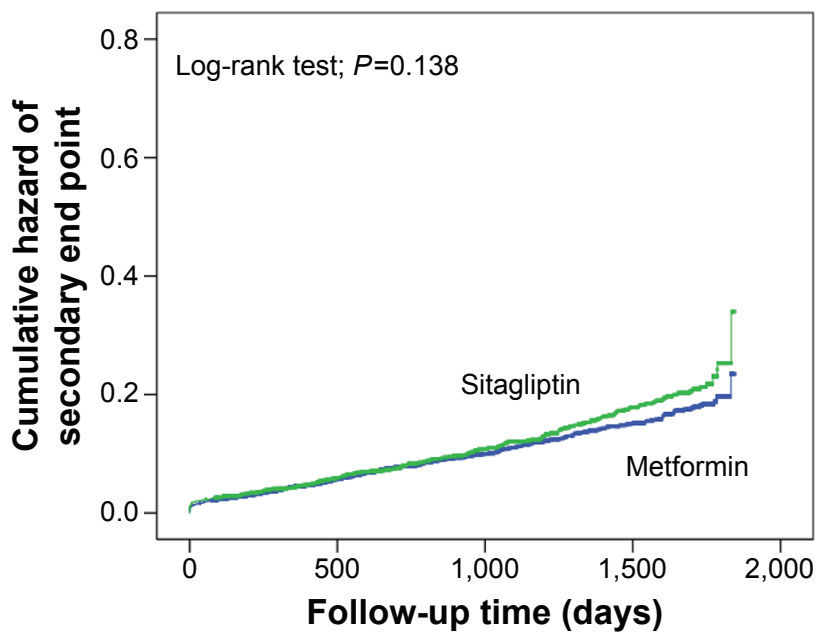

Figure 2 Kaplan-Meier curves for cumulative hazard of a secondary composite end point.

Note: Sitagliptin and metformin cohorts are propensity score-matched.




Table 4 Propensity score-matched analysis for safety end points

\begin{tabular}{llll}
\hline End point & Sitagliptin $\mathbf{( N = 1 , 6 2 0 ) , ~} \mathbf{n}(\%)$ & Metformin (N=3,240), n (\%) & P-value* \\
\hline Hospitalization for hypoglycemia & $10(0.6)$ & $16(0.5)$ & 0.677 \\
Hypoglycemia & $25(1.5)$ & $45(1.4)$ & 0.702 \\
Creatinine $>6.0 \mathrm{mg} / \mathrm{dL}$ or new-onset renal disease & $5(0.3)$ & $10(0.3)$ & 1.000 \\
Any pancreatitis & $10(0.6)$ & $12(0.4)$ & 0.258 \\
$\quad$ Acute & $3(0.2)$ & $8(0.2)$ & 0.761 \\
$\quad$ Chronic & $7(0.4)$ & $4(0.1)$ & 0.050 \\
Pancreatic cancer & $2(0.1)$ & $4(0.1)$ & 1.000 \\
\hline
\end{tabular}

Note: ${ }^{*}$-values were calculated with the use of Fisher's exact test.

minimal clinical relevance. Overall, there were no significant between-group differences in rates of the following adverse safety events: hospitalization for hypoglycemia, any hypoglycemic episode with blood glucose level $<54 \mathrm{mg} / \mathrm{dL}$, serum creatinine level $>6 \mathrm{mg} / \mathrm{dL}$, new-onset renal disease, and incident pancreatitis or pancreatic cancer.

\section{Discussion}

The adverse CV signal with DPP-4 inhibition was initially brought forth by the SAVOR-TIMI 53 study which suggested a possible link between the use of saxagliptin, the second DPP-4 inhibitor approved in 2009, and heart failure. ${ }^{14}$ The present study sought to ascertain the CV safety profile of sitagliptin, the first DPP-4 inhibitor with the largest share in prescription volume among the new class of antidiabetics, ${ }^{21}$ in Korean patients with T2DM. We used metformin-based therapy as our low-risk comparator, instead of placebo, to reflect real-world prescription practices and to ensure that adequate glycemic regulation was provided for patients in both intervention groups to avoid potential confounding due to intergroup differences in glucose control. This approach was also to ensure a more conservative estimate of potential $\mathrm{CV}$ risk because metformin has well-documented beneficial effects in CVD management via multiple proposed mechanisms, including improved insulin resistance, weight loss, reduction of total and low-density lipoprotein cholesterol, and by lowering the metabolic syndrome. ${ }^{23-27}$ The present study did not find any trend toward elevated CV risk associated with sitagliptin as compared to metformin. Additionally, sitagliptin was not associated with an elevated risk of heart failure. The rates of hypoglycemia and new-onset pancreatic disease were also comparable between the two groups. Our findings were consistent with the results from the only-available RCT with $\mathrm{CV}$ end points prespecified as primary outcomes to date, albeit one with placebo-controlled design. ${ }^{15}$ Based on the evidence available thus far, the presumed cardiac risk linked to saxagliptin appears to be at least not a class effect of DPP-4 inhibition.
The paucity of data on long-term CV risk with OAs is attributable to the conventional drug approval process set forth by the FDA, which was solely based on the effect of an agent on surrogate end points, such as serum glucose and $\mathrm{HbA}_{1 \mathrm{c}}$ reduction..$^{28}$ As a result, evaluation of more clinically relevant outcomes, such as CVDs, was not required in previous clinical trials designed for drug approval, nor was whether improved glycemic indexes lead to favorable $\mathrm{CV}$ outcomes in the long term. This was the case with sitagliptin which was approved prior to the 2008 FDA guidance regarding assurance of CV safety of all new glucose-lowering therapies. ${ }^{12}$ Sitagliptin was not subject to close scrutiny for $\mathrm{CV}$ risk, and hence the lack of clinical evidence supporting its $\mathrm{CV}$ effects up until the 2015 study by Green et al. The recent sitagliptin trial reported that it was not associated with excess risk of ischemic cardiac complications in patients with diabetes. ${ }^{15}$ However, the study was not without limitations. The placebo-controlled design may contribute to imbalances in background hypoglycemic treatment that could have influenced the CV outcomes. Moreover, as depicted in the Supplementary materials, the proportion of patients who were newly initiated on chronic insulin therapy or additional hypoglycemic therapy was greater in the placebo group compared to the sitagliptin group $(9.7 \%$ versus $13.2 \%, P<0.001$ and $21.7 \%$ versus $27.9 \%, P<0.001$, respectively). ${ }^{15}$ These intergroup differences in antidiabetic use may have substantial implications for the management of diabetes and CVDs, and thus could also have biased the results. To control such confounding, in the present study, we excluded those patients who received insulin, incretinbased therapy including DPP-4 inhibitors (except sitagliptin), or thiazolidinediones either during the preceding 6 months prior to cohort entry or at any time during the study period. However, despite these limitations, the 2015 study is the only available RCT and most relevant study in the sitagliptin-CV safety debate. As of today, no single RCT reproduced the unforeseen findings of the SAVOR-TIMI 53 study that suggested that DPP-4 inhibition might be associated with increased risk of heart failure. 
The debate on the cardiac safety of diabetes therapy originates from the controversial findings of the highly publicized 2007 meta-analysis by Nissen and Wolski. ${ }^{9}$ Due to the rare occurrence of $\mathrm{CV}$ events in individual trials, no study found a significant association between rosiglitazone and acute MI. The authors pooled data from 42 RCTs to boost the statistical power of data analysis and found a $43 \%$ increase in risk of MI and a marginally significant increase in risk of CV mortality among patients with diabetes who received rosiglitazone. ${ }^{9}$ These results and the findings from a series of observational studies over the subsequent years led to the 2010 imposition of substantial market restrictions by the FDA on the availability of the medication in treating type 2 diabetes. ${ }^{28-30}$ However, the validity of the 2007 metaanalysis has later been questioned for heterogeneity across included trials and the statistical methods that may have created misleading conclusions with regard to the drug's safety. ${ }^{29,31,32}$ Additionally, the data source may not have been appropriate for the intended evidence synthesis as most of the included studies did not have CVDs prespecified as primary end points but only analyzed them as safety end points. ${ }^{29}$ In 2013, in a surprising move, the FDA lifted the restrictions on prescription of rosiglitazone based on reassurance from postmarketing study findings. . $^{33,34}$

The rosiglitazone experience has many implications for selecting therapeutic options for treating diabetes as well as for preventing secondary CV complications. Apart from MI, the OA has been associated with various unexpected side effects (including edema, heart failure, lipid profile changes, weight gain, and fractures), ${ }^{7,83}$ many of which surfaced in postmarketing and surveillance studies. Although this medication is highly efficacious in glycemic control, the problem lies in its property of nonspecific binding to the gamma isoform of the peroxisome proliferator-activated receptor and our lack of understanding about the vast array of non-glycemic-related genes affected by the activation of peroxisome proliferator-activated receptor gamma, ${ }^{35}$ which could be at the root of its unexpected side effects. This raises the following question: how much do we know about DPP-4 inhibition? DPP-4 inhibitors enhance glucose-dependent insulin secretion by increasing the circulating concentration of bioactive incretins, such as glucagon-like peptide-1 and glucose-dependent insulinotropic peptide. ${ }^{36}$ Selective inhibition of DPP-4 may be essential for ensuring optimal safety of long-term therapy with this class of antidiabetics. Sitagliptin is a highly selective, competitive inhibitor with a high-affinity binding to the DPP-4 target. ${ }^{36}$ Clinical studies thus far have shown that sitagliptin was efficacious and well tolerated in patients with diabetes, with similar incidence rates of adverse events between the exposed and unexposed groups. However, as a relatively new OA with a favorable track record over less than a decade since its approval, more safety data on its long-term use need to be compiled through postmarketing and surveillance studies in order to synthesize compelling evidence on its effects in the diabetes management as well as secondary CVD prevention.

In the midst of the global diabetes epidemic, with Asia at its epicenter, ${ }^{37}$ the market for antidiabetic therapy has witnessed steady uptake in prescription volume over the last decade. Several new orally active antihyperglycemic agents became available for clinical use and underwent an immense growth in market share, especially DPP-4 inhibitors. ${ }^{21}$ A key driving force for antidiabetic demand is the growing prevalence of diabetes worldwide, particularly in Asia. ${ }^{37}$ To achieve optimal glycemic control in patients with diabetes, it is crucial to have a broad range of ammunition in our therapeutic arsenal as most patients with T2DM require an add-on therapy with a second or third OA. It appears that Asian patients have a tendency to develop diabetes at a younger age with a relatively lower BMI and are also at increased risk of diabetic complications, ${ }^{22}$ although the interethnic differences in the underlying pathophysiology of T2DM, beta-cell properties, genetic predisposition, and the pattern of diabetic complications are yet to be explained. ${ }^{37}$ Evidence is building up that Asian patients may benefit more from DPP-4 inhibitors than non-Asian patients, in that the agents appear to be more efficacious in achieving glucose homeostasis in patients with a lower BMI, a greater amount of visceral fat at a given BMI, and a predominant insulin secretory defect, the characteristics more prominent in Asian patients than in patients belonging to other ethnic groups. ${ }^{22,37-39}$ In a series of clinical trials, sitagliptin has proven to be effective for lowering not only FPG along with $\mathrm{HbA}_{1 \mathrm{c}}$ but also postprandial plasma glucose, which is additionally an important marker for predicting $\mathrm{CV}$ morbidity and mortality. ${ }^{39,40}$ Sitagliptin is also known for its relatively favorable tolerability profile: lower risk of chronic heart failure and edema relative to thiazolidinediones, lower risk of weight gain and hypoglycemia relative to sulfonylureas, lower risk of gastrointestinal intolerance and diarrhea relative to metformin, and a low propensity for drug interactions with other OAs. ${ }^{41-45}$ This present study also supported that, overall, sitagliptin is safe from the cardiac standpoint, and when used as a combotherapy with other hypoglycemic agents, it does not compromise patient CV safety and tolerability while contributing to achieving glucose homeostasis in patients with type 2 diabetes. 


\section{Limitations}

This study has limitations. Due to the retrospective and single-institution study design, our findings may not be applicable to patient population in other Asian countries. Although patient data were retrieved from institutional electronic medical records, diagnostic ICD-10 codes not entered or entered incorrectly by clinicians could have influenced the frequency of comorbid conditions and incidence rates of end-point events analyzed in our study. We did not include laboratory values that were not available from electronic medical records in our assessment for baseline clinical characteristics of the study patients such that missing data have no effect on the overall baseline distribution. The rarity of certain adverse events, such as pancreatitis and pancreatic cancer, along with a relatively small sample size may have contributed to a false negative result that failed to capture a potential significant association between the event and drug exposure. It was assumed that all prescriptions at discharge were dispensed and that the whole course of therapy was completed by patients, although individual patient compliance cannot be guaranteed. The clinically important question of whether improved glycemic regulation leads to reducing $\mathrm{CV}$ complications in patients with diabetes still remains to be answered. This study was not designed to answer the question as patient glucose levels in both intervention groups were relatively well controlled over the follow-up period with adequate antidiabetic therapy. We did not assess any special nutritional requirements that may have affected glycemic control of those patients treated with sitagliptin. However, to avoid including patients in acute conditions or in nothing by mouth status, we excluded those patients who received insulin either during the 6-month baseline period or at any time during the study period. An assumption was also made that any patients receiving orally administered antidiabetic medications had at least some nutritional intake.

\section{Conclusion}

This study suggested that sitagliptin was not associated with elevated risk of $\mathrm{CV}$ mortality and morbidity including MI, ischemic stroke, heart failure, and coronary revascularization, relative to metformin therapy among patients with type 2 diabetes in Korea. Similar results were found when only high-risk patients with an established CVD or $\mathrm{CV}$ risk factors were included for the subgroup analysis. Sitagliptin can be used as a viable add-on treatment for glucose homeostasis without compromising CV safety in patients with T2DM.

\section{Acknowledgments}

This study was supported by the Ajou University research fund and the Bio and Medical Technology Development Program of the National Research Foundation funded by the Ministry of Science, Information and Communications Technology, and Future Planning, Republic of Korea (No 2013M3A9B5075838).

\section{Disclosure}

The authors report no conflicts of interest in this work.

\section{References}

1. Gerstein HC, Pogue J, Mann JFE, et al. The relationship between dysglycaemia and cardiovascular and renal risk in diabetic and non-diabetic participants in the HOPE study: a prospective epidemiological analysis. Diabetologia. 2005;48(9):1749-1755.

2. Selvin E, Marinopoulos S, Berkenblit G, et al. Meta-analysis: glycosylated hemoglobin and cardiovascular disease in diabetes mellitus. Ann Intern Med. 2004;141(6):421-431.

3. Laakso M. Diabetes as a 'cardiovascular disease equivalent': implications for treatment. Nat Clin Pract Cardiovasc Med. 2008;5(11) 682-683.

4. Action to Control Cardiovascular Risk in Diabetes Study Group, Gerstein HC, Miller ME, et al. Effects of intensive glucose lowering in type 2 diabetes. $N$ Engl J Med. 2008;358(24):2545-2559.

5. Jørgensen CH, Gislason GH, Andersson C, et al. Effects of oral glucoselowering drugs on long term outcomes in patients with diabetes mellitus following myocardial infarction not treated with emergent percutaneous coronary intervention - a retrospective nationwide cohort study. Cardiovasc Diabetol. 2010;9:54.

6. Simpson SH, Majumdar SR, Tsuyuki RT, Eurich DT, Johnson JA. Doseresponse relation between sulfonylurea drugs and mortality in type 2 diabetes mellitus: a population-based cohort study. CMAJ. 2006;174(2): 169-174.

7. Delea TE, Edelsberg JS, Hagiwara M, Oster G, Phillips LS. Use of thiazolidinediones and risk of heart failure in people with type 2 diabetes: a retrospective cohort study. Diabetes Care. 2003;26(11):2983-2989.

8. Masoudi FA, Inzucchi SE, Wang Y, Havranek EP, Foody JM, Krumholz HM. Thiazolidinediones, metformin, and outcomes in older patients with diabetes and heart failure: an observational study. Circulation. 2005;111(5):583-590.

9. Nissen SE, Wolski K. Effect of rosiglitazone on the risk of myocardial infarction and death from cardiovascular causes. $N$ Engl J Med. 2007;356(24):2457-2471.

10. Holman RR, Paul SK, Bethel MA, Matthews DR, Neil HA. 10-Year follow-up of intensive glucose control in type 2 diabetes. $N$ Engl J Med. 2008;359(15):1577-1589.

11. American Diabetes Association. Standards of medical care in diabetes 2015. Diabetes Care. 2015;38 Suppl:S1-S93.

12. Guidance for industry: diabetes mellitus - evaluation cardiovascular risk in new antidiabetic therapies to treat type 2 diabetes. US Food and Drug Administration; 2008. Available from: http://www.fda.gov/ downloads/Drugs/GuidanceComplianceRegulatoryInformation/Guidances/ucm071627.pdf. Accessed January 15, 2016.

13. White WB, Cannon CP, Heller SR, et al. Alogliptin after acute coronary syndrome in patients with type 2 diabetes. NEngl J Med. 2013;369(14) 1327-1335.

14. Scirica BM, Bhatt DL, Braunwald E, et al. Saxagliptin and cardiovascular outcomes in patients with type 2 diabetes mellitus. $N$ Engl J Med. 2013;369(14):1317-1326

15. Green JB, Bethel MA, Armstrong PW, et al. Effect of sitagliptin on cardiovascular outcomes in type 2 diabetes. N Engl J Med. 2015;373(3): $232-242$. 
16. Udell JA, Cavender MA, Bhatt DL, Chatterjee S, Farkouh ME, Scirica BM. Glucose-lowering drugs or strategies and cardiovascular outcomes in patients with or at risk for type 2 diabetes: a metaanalysis of randomised controlled trials. Lancet Diabetes Endocrinol. 2015;3(5):356-366.

17. Frederich R, Alexander JH, Fiedorek FT, et al. A systematic assessment of cardiovascular outcomes in the saxagliptin drug development program for type 2 diabetes. Postgrad Med. 2010;122(3):16-27.

18. Monami M, Ahrén B, Dicembrini I, Mannucci E. Dipeptidyl peptidase-4 inhibitors and cardiovascular risk: a meta-analysis of randomized clinical trials. Diabetes Obes Metab. 2013;15(2):112-120.

19. Scheen AJ. Cardiovascular effects of gliptins. Nat Rev Cardiol. 2013;10(2):73-84.

20. Kim SC, Glynn RJ, Liu J, Everett BM, Goldfine AB. Dipeptidyl peptidase-4 inhibitors do not increase the risk of cardiovascular events in type 2 diabetes: a cohort study. Acta Diabetol. 2014;51(6):1015-1023.

21. Hampp C, Borders-Hemphill V, Moeny DG, Wysowski DK. Use of antidiabetic drugs in the US, 2003-2012. Diabetes Care. 2014;37(5): 1367-1374.

22. Kim YG, Hahn S, Oh TJ, Kwak SH, Park KS, Cho YM. Differences in the glucose-lowering efficacy of dipeptidyl peptidase-4 inhibitors between Asians and non-Asians: a systematic review and meta-analysis. Diabetologia. 2013;56(4):696-708.

23. Herman WH, Edelstein SL, Ratner RE, et al. Effectiveness and costeffectiveness of diabetes prevention among adherent participants. Am J Manag Care. 2013;19(3):194-202.

24. Diabetes Prevention Program Research Group. Long-term safety, tolerability, and weight loss associated with metformin in the Diabetes Prevention Program Outcomes Study. Diabetes Care. 2012;35(4):731-737.

25. Johnson JA, Simpson SH, Toth EL, Majumdar SR. Reduced cardiovascular morbidity and mortality associated with metformin use in subjects with Type 2 diabetes. Diabet Med. 2005;22(4):497-502.

26. Fontbonne A, Charles MA, Juhan-Vague I, et al. The effect of metformin on the metabolic abnormalities associated with upper-body fat distribution. BIGPRO Study Group. Diabetes Care. 1996;19(9):920-926.

27. Kitabchi AE, Temprosa M, Knowler WC, et al. Role of insulin secretion and sensitivity in the evolution of type 2 diabetes in the diabetes prevention program: effects of lifestyle intervention and metformin. Diabetes. 2005;54(8):2404-2414.

28. Hiatt WR, Kaul S, Smith RJ. The cardiovascular safety of diabetes drugs - insights from the rosiglitazone experience. N Engl J Med. 2013; 369(14):1285-1287.

29. Rawson NS. Review of the quality of observational studies of the association between rosiglitazone and acute myocardial infarction. J Popul Ther Clin Pharmacol. 2014;21(2):e214-e232.

30. FDA Drug Safety Communication: updated risk evaluation and mitigation strategy (REMS) to restrict access to rosiglitazone-containing medicines including Avandia, Avandamet, and Avandaryl. US Food and Drug Administration; 2011. Available from: http://www.fda.gov/ Drugs/DrugSafety/ucm255005.htm. Accessed January 15, 2016.
31. Friedrich JO, Beyene J, Adhikari NK. Rosiglitazone: can meta-analysis accurately estimate excess cardiovascular risk given the available data? Re-analysis of randomized trials using various methodologic approaches. BMC Res Notes. 2009;2:5.

32. Diamond GA, Bax L, Kaul S. Uncertain effects of rosiglitazone on the risk for myocardial infarction and cardiovascular death. Ann Intern Med. 2007;147(8):578-581.

33. FDA requires removal of certain restrictions on the diabetes drug Avandia. US Food and Drug Administration; 2013. Available from: http://www.fda.gov/NewsEvents/Newsroom/PressAnnouncements/ ucm376516.htm. Accessed January 15, 2016.

34. Home PD, Pocock SJ, Beck-Nielsen H, et al. Rosiglitazone evaluated for cardiovascular outcomes in oral agent combination therapy for type 2 diabetes (RECORD): a multicentre, randomised, open-label trial. Lancet. 2009;373(9681):2125-2135.

35. Kung J, Henry RR. Thiazolidinedione safety. Expert Opin Drug Saf. 2012;11(4):565-579.

36. Thornberry NA, Gallwitz B. Mechanism of action of inhibitors of dipeptidyl-peptidase-4 (DPP-4). Best Pract Res Clin Endocrinol Metab. 2009;23(4):479-486.

37. Ma RC, Chan JC. Type 2 diabetes in East Asians: similarities and differences with populations in Europe and the United States. Ann N Y Acad Sci. 2013;1281:64-91.

38. Aso Y, Ozeki N, Terasawa T, et al. Serum level of soluble CD26/ dipeptidyl peptidase-4 (DPP-4) predicts the response to sitagliptin, a DPP-4 inhibitor, in patients with type 2 diabetes controlled inadequately by metformin and/or sulfonylurea. Transl Res. 2012;159(1):25-31.

39. Nakagami T; DECODA Study Group. Hyperglycaemia and mortality from all causes and from cardiovascular disease in five populations of Asian origin. Diabetologia. 2004;47(3):385-394.

40. Deacon CF. Dipeptidyl peptidase-4 inhibitors in the treatment of type 2 diabetes: a comparative review. Diabetes Obes Metab. 2011; 13(1):7-18.

41. Ahrén B. Emerging dipeptidyl peptidase-4 inhibitors for the treatment of diabetes. Expert Opin Emerg Drugs. 2008;13(4):593-607.

42. Phung OJ, Scholle JM, Talwar M, Coleman CI. Effect of noninsulin antidiabetic drugs added to metformin therapy on glycemic control, weight gain, and hypoglycemia in type 2 diabetes. JAMA. 2010;303(14): $1410-1418$.

43. Pratley RE, Salsali A. Inhibition of DPP-4: a new therapeutic approach for the treatment of type 2 diabetes. Curr Med Res Opin. 2007;23(4): 919-931.

44. Williams-Herman D, Round E, Swern AS, et al. Safety and tolerability of sitagliptin in patients with type 2 diabetes: a pooled analysis. $B M C$ Endocr Disord. 2008;8:14.

45. Gallwitz B. Sitagliptin: profile of a novel DPP-4 inhibitor for the treatment of type 2 diabetes. Drugs Today (Barc). 2007;43(1):13-25.
Therapeutics and Clinical Risk Management

\section{Publish your work in this journal}

Therapeutics and Clinical Risk Management is an international, peerreviewed journal of clinical therapeutics and risk management, focusing on concise rapid reporting of clinical studies in all therapeutic areas, outcomes, safety, and programs for the effective, safe, and sustained use of medicines. This journal is indexed on PubMed Central, CAS,
Dovepress

EMBase, Scopus and the Elsevier Bibliographic databases. The manuscript management system is completely online and includes a very quick and fair peer-review system, which is all easy to use. Visit http://www.dovepress.com/testimonials.php to read real quotes from published authors. 